\title{
Determinantes socioculturales de la politica alimentaria: voces de las personas beneficiarias del programa Comedores Comunitarios. Estudio realizado en la Ciudad de Culiacán Sinaloa México
}

\section{Sociocultural determinants of food policy: voices of the beneficiaries of the Community kitchens program. Study in the city of Culiacan Sinaloa Mexico}

\author{
LUZ MERCEDES VERDUGO ARAUJO \\ Docente investigadora del Programa de Licenciatura en Trabajo Social de la Universidad Autónoma de Sinaloa. (México) \\ luzmercedesverdugo@hotmail.com / https://orcid.org/0000-0002-0589-9778
}

MARTHA LETICIA CABELLO GARZA

Docente investigadora del Centro De Investigación y Desarrollo en

Ciencias de la Salud de la Universidad Autónoma de Nuevo León. (México)

marthacabello1@gmail.com / https://orcid.org/0000-0001-6580-9650

\begin{abstract}
Resumen. El propósito de este trabajo es dar a conocer parte de los resultados de una investigación sobre el proceso de implementación de una política social alimentaria a través de Comedores Comunitarios en atención a la inseguridad alimentaria en la ciudad de Culiacán, Sinaloa, México. El objetivo fue conocer los elementos socioculturales que determinan la eficiencia del programa social. El diseño de la investigación es descriptivo-interpretativo utilizando un enfoque cualitativo basado en un método hermenéutico-critico que recupera los discursos de 30 personas beneficiadas de 27 Comedores Comunitarios que operan el programa en la Ciudad de Culiacán, Sinaloa, México. Las representaciones del proyecto político significan para las personas beneficiarias una opción de alimentación y crean un espacio de convivencia y descanso. Los discursos muestran lo que viven y sienten respecto a la cotidianidad del comensal y son reflejados a través de 6 dimensiones de determinantes socioculturales: dimensión selectiva, resistencia cultural, dependencia, vulnerabilidad de salud, ecológico-social y el vacío del proyecto familiar, los cuales precisan en la acción lo cultural y político. Asimismo se visualiza la necesidad de conocer las determinantes socioculturales de la alimentación para contribuir a transformar la realidad alimentaria, y reconocer los factores estructurales de la inseguridad alimentaria, pero también las condicionantes en el seno de las prácticas sociales de las familias, ya que ambas son importantes a la hora de tomar decisiones de la política alimentaria.
\end{abstract}

Palabras clave: política alimentaria, determinantes socioculturales de la alimentación, inseguridad alimentaria, comedores comunitarios. 


\begin{abstract}
The purpose of this paper is to present some of the results of a research into the process of implementing of a social food policy through Community Kitchens in response to food insecurity in the city of Culiacan, Sinaloa, Mexico. The objective was to know the sociocultural factors that determine the efficiency of this social program. The research design is descriptive-interpretive using a qualitative approach based on a hermeneutic-critical method that recovers the discourses of 30 people benefited of 27 Community Kitchens who operate the program in the City of Culiacan, Sinaloa, Mexico. The representations of the political project mean for people benefiting a feeding option, and create a space for conviviality and rest. The discourses show what they live and feel with regard to daily life of the diner and are reflected through six dimensions of sociocultural determinants: selective dimension, cultural resistance, dependency, health vulnerability, ecological-social and the emptiness of the family project, which require cultural and political action. Likewise, the need to know the sociocultural determinants of food is visualized, in order to contribute to transforming the food reality, and to recognize the structural factors of food insecurity, but also the determining factors within the social practices of families, since both are important when making food policy decisions.
\end{abstract}

Key words: food policy, sociocultural determinants of food, food insecurity, community kitchens.

\section{INTRODUCCIÓN}

El análisis de la política alimentaria tradicionalmente, ha sido abordado desde métodos lineales que no han dado respuesta a la situación de inseguridad alimentaria. Muestra de ello son los modelos verticales de política social, donde las fases siguen una lógica estructural y reflejan falta de sinergia entre ellas. Lo anterior no ha servido para atenuar la inseguridad alimentaria al contrario las cifras estadísticas demuestran que el problema sigue en aumento. Esta situación se observa al analizar los indicadores de obesidad, sobre todo en México que tiene una tasa del $33 \%$ en obesidad de adultos, ocupando la segunda más alta en la Organización para la Cooperación del Desarrollo Económico (OCDE, 2017), mucho mayor que el promedio de los países que la componen que es de $19.4 \%$. Además, 35\% de los adolescentes de 12 a 19 años de edad tienen sobrepeso u obesidad.

De acuerdo con las últimas estimaciones de la Organización de las Naciones Unidas para la Alimentación y la Agricultura (2019), en el mundo hay 820 millones de personas crónicamente subalimentadas. Así mismo el Consejo Nacional de Evaluación de la Política de Desarrollo Social (CONEVAL, 2015), en el año 2014 estimó que 55.3 millones de personas en México vivían en pobreza y 28 millones (23.4\%) presentaban carencia por acceso a la alimentación, es decir, no tenían acceso a alimentos suficientes, inocuos y nutritivos.

Esta situación obliga a modificar el enfoque de la política social alimentaria desde los marcos de diseño de implementación y evaluación, que han resultado insuficientes para atender la compleja realidad alimentaria. En el caso de la política alimentaria los evaluadores para medir la eficacia de los programas sociales lo hacen con el impacto en la seguridad alimentaria. Esto lo han realizado desde una visión economista, utilizando los indicadores de acceso y disponibilidad, dejando de lado otros aspectos que determinan la alimentación en los grupos sociales. La mayoría de las personas expertas coinciden en que una dieta saludable y sostenible integra, por un lado, qué comemos, pero por otro, cómo comemos, como nos proveemos de los alimentos y como los cocinamos (Molero, López y Arroyo, 2018). Debido a lo anterior una nueva visión de política alimentaria debe conocer las prácticas alimentarias, así como analizar la relación entre el sistema social, los modos de vivir y la salud.

Hoy en día, las políticas de salud proponen combatir la carencia alimentaria con políticas coordinadas para que las familias tengan acceso a alimentos inocuos y nutritivos. Para Yaschine, Ochoa y Hernández (2014), se está enfatizando en incorporar la participación social desde el diseño, ejecución, evaluación y retroalimentación de los programas sociales. Una de las principales estrategias que está operando en México para atenuar la inseguridad alimentaria es el programa Comedores Comunitarios, objeto de análisis de la presente investigación. Un estudio encontrado sobre el tema de los comedores comunitarios en Sonora, México (Arellano y Álvarez, 2019) reflejan el desfase entre las políticas alimentarias y las discusiones actuales sobre alimentación saludable, además analiza la voluntad política hacia una visión del desarrollo sostenible centrado en los derechos sociales. 
La Secretaria de Desarrollo Social (SEDESOL, 2015) señala que el objetivo general del programa Comedores Comunitarios es desarrollar, fortalecer y consolidar una estrategia de atención alimentaria y nutricional, proporcionando alimentos nutritivos de calidad y cantidad suficientes a grupos poblacionales con problemas de desnutrición, en riesgo de padecerla y en inseguridad alimentaria, a partir del fomento de la participación comunitaria, y con ello contribuir a abatir la carencia por acceso a la alimentación.

Por lo tanto es necesario analizar si realmente el programa abate la inseguridad alimentaria, o si realmente considera las determinantes contextuales y socioculturales de la alimentación por ser la principal estrategia que opera en el país. Por lo que es necesario conocer las voces de las personas beneficiadas del programa social pues son los implicados directos donde recaen las acciones de esta política. Sin embargo, una vez atendido el plano individual, este cobra sentido en lo colectivo con plena conciencia de lo que se busca mejorar. Por lo que de acuerdo a Verdugo-Araujo, Tereso-Ramírez y CarriIlo-Montoya (2019), se debe dialogar con otros actores y con otros niveles de estructuras a fin de beneficiar a la población mas vulnerable.

Para Cabello y Hernández (2016), los nuevos modelos de intervención deben atender las especificidades regionales y locales como un elemento clave de la acción profesional en trabajo social, ya que no se pueden seguir aplicando recetas universales para necesidades particulares ignorando las condiciones del aquí y el ahora. Tomando en cuenta estos elementos se estaría rompiendo con los esquemas tradicionales con que ha operado la política alimentaria. El trabajo social puede favorecer el desarrollo de una política social en la medida en que contribuya a la superación de los obstáculos que se interponen en su desenvolvimiento $y$, en la medida en que se elaboren líneas de intervención que posibiliten mayor integración y bienestar social.

\section{POLÍTICA ALIMENTARIA Y DETERMINANTES SOCIOCULTURALES DE LA ALIMENTACIÓN}

El objetivo central de las políticas alimentarias en su devenir histórico ha sido desarrollar estilos de vida saludables y se refieren a las directrices y programas relacionados con la disponibilidad, el acceso, la distribución y la utilización de los alimentos. Se puede precisar, que son el conjunto de medidas y programas desarrollado por los Estados con la finalidad de garantizar el derecho a la alimentación a través de la suficiencia, accesibilidad física y económica y calidad de los alimentos que permita prevenir y controlar los estados de malnutrición por déficit y por exceso, garantizando el buen estado de salud a individuos y grupos poblacionales, con énfasis en los más vulnerables y atendiendo a todos los niveles de la sociedad (Díaz y Candela, 2014).

Analizando las concepciones sobre seguridad alimentaria, Mundo, Shamah-levy y Rivera-Domarco (2013), señalan que ésta se presenta cuando las personas tienen en todo momento acceso físico y económico a suficientes alimentos inocuos y nutritivos para satisfacer sus necesidades y sus preferencias, a fin de llevar una vida activa y sana. Por el contrario, un acceso nulo o incierto a los alimentos se ha definido como inseguridad alimentaria (Cabello y Amador, 2015).

Se observa en las dimensiones de la seguridad alimentaria de la FAO que no contempla las determinantes socioculturales en la alimentación, así como las cuestiones subjetivas del consumo. Dicho enfoque es para evaluar la seguridad alimentaria desde la estructura, desde lo nacional, pero no abarca el contexto de la seguridad alimentaria en las familias, en el espacio de significaciones sociales. Cabe señalar que dichas dimensiones son las que se consideran para medir la efectividad de las políticas alimentarias; en la medida en que se cumplan dichas dimensiones, se mide el impacto, por lo que no es de extrañarse por qué las políticas alimentarias no contienen elementos de los espacios donde recaen sus acciones.

La realidad alimentaria se puede definir desde la postura de Hibridación Alimentaria, la cual es un panorama de fusión y apropiación de bienes materiales y simbólicos de consumo. Toda vez que las prácticas de alimentación expresan y configuran nuevas formas de ordenamiento social, que inciden en los comportamientos e interacciones de los sujetos, en el ámbito individual, familiar, grupal y social. Para García (1989) las hibridaciones culturales son los modos en que determinadas formas se van separando de prácticas existentes para recombinarse en nuevas formas y nuevas prácticas. En las condiciones de globalización actuales, el empleo del concepto de hibridación parece más útil para nombrar esas mezclas en las que no sólo se combinan elementos étnicos o religiosos, sino que se intersectan con productos de las tecnologías avanzadas y el proceso de industrialización alimentaria.

En este sentido analizar la problemática alimentaria para Sordini (2016) no solo es abordarla desde la producción, distribución y comercialización sino también abarcar desde una cuestión social es decir tomar en cuenta los aspectos vinculados al consumo y sus efectos sobre las condiciones históricas de reproducción de la población y de la fuerza de trabajo, una de cuyas expresiones es la situación nutricional de vastos sectores sociales. Por lo tanto puede hablarse de un acceso 
diferencial a los alimentos como resultado de un acceso diferencial de distribución del producto social entre los distintos agentes sociales.

Para Hurtado (2019), la seguridad alimentaria y nutricional, el derecho a la alimentación y la soberanía alimentaria son tres aristas fundamentales para la solución de problemas de alimentación, este autor concluye que las debilidades en la comprensión de estos abordajes ha generado dificultades en el diálogo entre los diversos actores relacionados a esta problemática, en este trabajo se presentan las voces de actores que son los beneficiarios directos del programa de comedores comunitarios.

Por otra parte un elemento importante que ha modificado las prácticas alimentarias es la incorporación de la mujer al mercado laboral, lo que ha repercutido en la transformación de la figura materna en la línea de no ser la que cambia la materia en el alimento y la coloca en la mesa del hogar, sino que se encuentra expuesta a recurrir a otras formas de crear y recrear la alimentación. Otro factor que introduce cambios socioculturales, es la gran distancia entre los hogares y los centros de trabajo, debido a que se desestructura la dieta familiar al modificarse los sitios, horarios de consumo y el número de integrantes que pueden comer juntos (Verdugo, 2012). Asimismo entre los factores culturales cabe destacar la manera en que las diversas etnias preparan los alimentos según las regiones de procedencia y ello tiene que ver con el tipo de alimento, la forma en que se consume y sus procedencias culturales.

Es importante que las intervenciones alimentarias retomen las dimensiones socioculturales en la alimentación para tener impacto en la salud de la población. Sus actuaciones deben ir acompañadas de investigaciones respecto a las prácticas alimentarias locales para evitar desconocer las características nutricionales del sistema alimentario de la población beneficiada (Carrasco, 2004). En función de lo anterior es necesario conocer ¿cuáles son los significados que tiene el comedor comunitario para las personas beneficiadas? ¿Cuáles son las determinantes socioculturales que determinan la eficiencia del programa social? y en función de esto ¿Qué factores debe tomar en cuenta la política alimentaria para una mejor intervención social?

\section{METODOLOGÍA}

El presente estudio descriptivo-interpretativo tiene un diseño cualitativo, considerando que la política alimentaria es parte de una realidad simbólica estructurada por significaciones y aspectos subjetivos que constituyen comportamientos sociales que como menciona Alguacil (2011), se preocupa por los aspectos simbólicos y subjetivos que constituyen comportamientos sociales y que mueven a la sociedad desde el punto de vista de los significados que los sujetos y los grupos dan a su vida en sociedad. Este enfoque trabaja el lenguaje y por tanto, los discursos expresan las cualidades que los sujetos dan a los fenómenos sociales que ellos mismos experimentan. Utiliza la conversación con la intención de profundizar en lo subyacente de los comportamientos sociales, ya que son los que construyen el mundo social.

Desde ese posicionamiento se utilizó el método hermenéutico crítico el cual considera que si bien el lenguaje es para fines de comprensión y comunicación, también es un medio de dominio y poder. Habermas (1987), considera que para comprender totalmente el objeto de la interpretación o lograr una comunicación no distorsionada con otros, no sólo deben emplearse principios hermenéuticos, sino recurrir además, a la explicación de las limitantes reales que actúan sobre el intérprete. En este sentido, Díaz (2007) señala que las representaciones y simbolizaciones contenidas en los códigos lingüísticos se exponen a un proceso fáctico de comprensión que se logra por medio de las palabras en los discursos que se generan en las relaciones, y es a través de esto que el lenguaje alcanza su sentido, es decir, la condición pragmática que lo orienta hacia un entendimiento que debe ser discutido, reflexionado y consensuado.

Como técnicas de recolección de datos este estudio utilizó la observación, con la idea de caracterizar el escenario, las interacciones de los actores, la comunicación no verbal y los distintos diálogos empleados en los discursos. Se utilizó la técnica de la entrevista semiestructurada como una forma de contacto cercano y directo con los actores sociales para establecer un diálogo comunicativo y participativo mediante el lenguaje, donde describan profundamente su experiencia vivida en el programa social, permitiendo describir las significaciones socioculturales de seguridad alimentaria a partir del desarrollo de la política.

Para el análisis de los datos se utilizó el análisis crítico de discurso usando la codificación de la información para una esquematización que permitió tener una visualización de las respuestas, acompañado de un análisis cualitativo hermenéutico, donde emergieron algunas categorías como significadores de la política alimentaria y como determinantes socioculturales de la alimentación.

Considerando a López y Deslauriers (2011), la codificación de la información, se realizó identificando las unidades de registro, que son las unidades de sentido que pueden estar en un mismo texto, en una misma palabra o en un mismo 
grupo de frases. En este trabajo se identificaron las unidades de registro, se les asignó un código que permitió identificar y clarificar las informaciones obtenidas en las entrevistas, relacionándolas con las categorías de la investigación.

Se recurrió a un muestreo no probabilístico de juicio, aplicando los instrumentos de recolección de datos a 30 personas beneficiadas de 27 Comedores Comunitarios de la Ciudad de Culiacán, Sinaloa. Uno de los criterios de inclusión fue que las personas tuvieran por lo menos un lapso mayor a seis meses asistiendo de forma constante al Comedor Comunitario. En la Tabla 1 se muestra el perfil de las personas participantes del estudio.

Cabe aclarar que previo a la recolección de datos, los sujetos de investigación fueron enterados de los objetivos de la investigación, todos ellos firmaron un consentimiento informado, donde se garantizó el anonimato y se especificó que la confidencialidad a la identidad de cada uno de los participantes sería respetada, además que las entrevistas y material quedarían siempre bajo resguardo de la investigación.

\section{RESULTADOS}

Los hallazgos muestran elementos que referencian los significados que tiene el comedor comunitario para las personas beneficiadas. Los datos encontrados durante la aplicación de las técnicas cualitativas muestran que las representaciones del proyecto político significan para el beneficiario una opción de alimentación y un trabajo comunitario donde una líder trabaja para la alimentación del lugar, creando un espacio de convivencia y descanso. Se muestran además narraciones de actores que viven y sienten los efectos de la política alimentaria en la cotidianidad del Comedor Comunitario, y un discurso de la mediadora comunitaria, que es la encargada del funcionamiento del espacio del Comedor Comunitario en la localidad y los discursos de los destinatarios finales de la política, que son los personas beneficiadas.

Las personas beneficiarias poseen en su figura un factor que delata la procedencia cultural que le proyecta los sentidos de respuesta ante el proceder de la política. El primer momento ante el escenario social es por un lado reconocerse carente de una necesidad y por el otro una expresión de vergüenza; es decir, se percibe como un grupo social que no alcanza a comprender el porqué de su permanencia en un Comedor Comunitario. La dimensión de este acontecimiento y las reacciones de asistir a consumir el alimento se refiere a "ser pobre" y esto lleva consigo un lenguaje social que muestra en sus manos y piel la dependencia del otro llamado gobierno para lograr la sobrevivencia alimentaria.

\begin{tabular}{|lc|}
\hline \multicolumn{2}{|c|}{ TABLA I. CARACTERISTICAS SOCIODEMOGRÁfICAS DE } \\
LAS PERSONAS PARTICIPANTES EN EL ESTUDIO \\
Sexo & \\
Femenino & 17 \\
Masculino & 13 \\
\hline Edad & \\
$20-30$ & 7 \\
$31-40$ & 9 \\
$41-50$ & 5 \\
$51-60$ & 4 \\
$61-m a s$ & 5 \\
\hline Nivel educativo & \\
Sin estudios & 6 \\
Primaria & 8 \\
Secundaria & 6 \\
Preparatoria & 9 \\
Carrera profesional (jubilada) & 1 \\
\hline Ocupación & \\
Ama de casa & 12 \\
Estudiante & 3 \\
Albañil & 3 \\
Técnico(a) u oficios & 10 \\
Desempleado & 1 \\
Jubilado & 1 \\
\hline
\end{tabular}

Fuente: elaboración propia.

Su reacción a la presencia de extraños delata intimidación, comiendo de manera rápida para marcharse cuanto antes e inclinando su cabeza como señal de vergüenza. Algunos expresan este sentimiento afirmando lo siguiente: “Es que le da pena a uno venir a comer aquí y que nos vean todos los que van pasado, diciendo mira aquí están comiendo los más jodidos" [ríe]. (E.13).

Sentirse como los sujetos más vulnerables de la política, los evidencia a la vista de todos, esto representa un acto de exclusión del desarrollo social. Según los discursos para algunas personas de la comunidad el acudir a sentarse ahí es una "expresión social de humillación". Para otras, se traduce en beneficio inmediato para las personas que trabajan cerca en instituciones, quienes afirman que lo visualizan "como una fonda" donde pueden adquirir alimentos a buen precio. Desde esta perspectiva, el proyecto que se implementa desdibuja el referente alimentario como un acto cultural e íntimo en el seno familiar y lo proyecta en un escenario panorámico que polariza la atención a las personas beneficiadas ante la visión comunitaria. Esta exposición proyecta significados socioculturales, que expone a los necesitados a lo público, confronta a lo íntimo del proceso de alimentación y visibiliza la significación de dicha acción que se convierte en la legitimación del proyecto político. 
En un segundo momento se encuentran las dimensiones culturales que limitan la actuación del programa alimentario. Por una parte, las determinantes socioculturales de la alimentación en el proyecto significan factores contextuales estructurales de la institución política; por otra, la procedencia de los espacios culturales y el individuo que presenta lo selectivo social de la alimentación y que se visualiza en los discursos de los actores. Los determinantes socioculturales reflejan dimensiones que precisan en la acción lo cultural y político. En consecuencia, crea espacios evaluativos del programa y detalla la respuesta en la acción.

En la investigación se encontraron seis dimensiones: la primera dimensión es selectiva, reafirma características de la cultura alimentaria; la segunda fase refiere la resistencia cultural de alimentación; la tercera, la dependencia regional-cultural; la cuarta, la vulnerabilidad de salud como determinante social en la alimentación; la quinta, lo ecológico social en la alimentación que radica en la supervisión del contexto y, la sexta, en el vacío del proyecto familiar, en la convivencia social.

Desde esta perspectiva, se trazan la dimensión cultural, entendiendo por cultura lo que Durham (1984) señala como dimensión simbólica de la sociedad presente en todas las prácticas y procesos sociales, y más explícitamente en los procesos de significación, de producción de sentido y de comunicación, donde los códigos o acuerdos sociales aparecen implícita o explícitamente. Está dimensión es verbalizada en el discurso, cristalizada en el mito, en el rito y en el dogma; incorporada a los artefactos, a los gestos y a la postura corporal. Desde esta mirada, los discursos representan los rasgos culturales y los grados de resistencia que constituyen asimismo los grados de impacto.

En estos grados de impacto, la intensidad de visibilidad se torna difusa, con lo cual el proyecto político presenta desfases en la acción al no considerar lo micro en los espacios sociales. En este sentido, Giménez (2005) señala que es importante retomar el espacio donde se establecen las interacciones sociales, pues es donde se encuentran los esquemas y representaciones compartidas y objetivadas en formas simbólicas en contextos históricamente específicos y socialmente estructurados. No considerar la dimensión subjetiva del contexto, resultaría en la invisibilidad; por tanto, se configura en determinantes socioculturales que se trazan en dimensiones de la cotidianidad de la política.

En la primera dimensión de la acción de lo cultural y lo político en torno a los espacios evaluativos del programa se encuentran características de los personas beneficiadas en cuanto a la línea de participación, en el cual se demarcan dimensiones socioculturales de la alimentación que determinan su asistencia y constancia al proyecto político. Desde este posicionamiento encontramos un discurso condicionado por las personas beneficiadas, los cuales abordan en su lenguaje social una serie de dimensiones socioculturales que los hacen no participar. Desde este abordaje se dibuja la primera determinante que refleja la dimensión de selección basada en su cultura alimentaria, que los lleva a decidir sobre qué alimentos desean recibir en el comedor. La líder demarca estas características al señalar que: "La gente es muy mañosa, oiga, no le gusta comer cualquier cosa, menos si es un caldo con verduras; ellos quieren un buen plato y rebosante; mandan preguntar qué habrá y depende de eso su participación. A veces los niños vienen y preguntan doña Lupe qué hizo de comer; les digo que sopa de verduras y contestan: avise cuando haga pozole" (E. 8).

Desde esa dimensión, se argumentan rasgos de selección de alimentación y los atributos que debe poseer el alimento de su agrado. Con este elemento se vislumbra que la participación de los personas beneficiadas está relacionada con el tipo de alimentos que se ofrecen; prefieren alimentos "Ilenadores a alimentos que ellos los denominan light" que hará que pronto tengan hambre: Nos gusta un alimento completo y llenador porque luego uno come un caldito muy ligero y le da hambre rápido; hay que estar fuerte, sobre todo cuando realizamos actividades muy pesadas y tenemos jornadas intensas en los hogares (E.4). Al respecto la mediadora comunitaria señala: Las personas que vienen a comer son personas que tienen jornadas de trabajo larga y busca venir al comedor como una comida de fácil acceso (E. 1).

Desde estos discursos se observa cómo los personas beneficiadas argumentan el porqué de su selección alimentaria; le dan sentido a sus preferencias culturales relacionándolas con las actividades de la vida cotidiana, dando a su vez un discurso de convencimiento para justificar sus actuaciones. En estos aspectos entra en juego una segunda determinante, que es la resistencia cultural de la alimentación. Esto radica en que consideran que los alimentos que seleccionan como parte de su dieta alimentaria no son los alimentos más sanos, sin embargo, no desean cambiarlos y los han adoptado como parte de su identidad alimentaria.

En esta dimensión entra en juego el proceso sujetos-sociedad. Esta articulación es mencionada por Giménez (2005), al señalar que existen sujetos que producen y sujetos que consumen. Estos últimos se apropian de lo producido reconfigurándolo o confiriéndoles un nuevo sentido. Esta afirmación explica las nuevas prácticas alimentarias: Es muy propositivo los alimentos que quieren ofrecer, pero a uno le gusta lo grasoso 
y pues buscamos que esos días que vamos a comer aquí puede ser que hagan algo al gusto del paladar (E.5). En este sentido la mediadora comunitaria comenta: La gente no es constante solo vienen a comer cuando les gusta lo que hay (E. 4).

Estas prácticas, a su vez, se transmiten a los niños más pequeños de las familias, pues la mayoría de los pequeños de la comunidad funge el papel de mensajero, el hilo conductor que entrelaza a la líder y a la madre de familia, el que corre al espacio a preguntar el menú del día para observar la forma en que su madre da la última palabra. La madre es la que decide si asistirán a degustar el alimento al comedor; es la que crea los lazos de dependencia al programa alimentario. Se puede deducir que esta dependencia al proyecto es una dependencia regional y cultural y de práctica familiar que detalla los rasgos de una tercera determinante que aborda las condiciones regionales. Se detallan rasgos culturales de alimentación de personas que habitan en un contexto denominado «la franja de oro» por la riqueza cultural de comida acostumbrados a degustar platillos vastos de alimentos y que entran en conflicto al recibir alimentos ligeros; se demarcan rasgos de un contexto productor de alimento y su relación con el consumo alimentario en los hogares.

Asimismo, entre los rasgos que se suman a la decisión de la madre de familia sobre la asistencia al comedor y tiene que ver con una cuarta determinante relacionada con la vulnerabilidad de salud donde se desprende la salud alimentaria caracterizada por la calidad sanitaria. La calidad del alimento depende de quién lo elabora; preferimos venir con la señora Lalita, ella siempre sirve buena ración y está limpio, pero con las otras no, porque siempre tienen lleno de moscas el lugar (E.6). Al respecto la mediadora comunitaria señala: Cuando me toca realizar la comida siempre tengo lleno porque me gusta mantener limpio el comedor, parece que no pero la gente se fija en todo (E.20).

Dentro de los valores de los alimentos que evalúan como atributos positivos está la higiene en la preparación de los alimentos, factor que las personas beneficiadas lo asocian a la salud. Por tanto, si el comedor presenta rasgos antihigiénicos ellos asumen que los alimentos están contaminados y preparados incorrectamente, lo cual representa un riesgo alimentario que marca vulnerabilidad social en la salud. La quinta dimensión aborda un aspecto importante, como lo es lo ecológico social, caracterizado por la supervisión del contexto, es decir, el espacio geográfico donde está situado el comedor: La verdad, no nos gusta venir a comer porque está a un lado el canal y en ocasiones las personas lo usan para tirar basura y animales; a veces están presentes muchas moscas y mosquitos (E.7).
La ubicación del comedor es muy importante porque establece condiciones de seguridad en el momento de comer en el espacio y da tranquilidad de que lo que ahí consumen no repercutirá en su salud. Asimismo, se traza otro elemento crucial relacionado con su participación: los precios variados de los alimentos: Es fácil acceder a la alimentación, ya que son buenos precios si se viene solo a comer, pero ya en familia se complica la situación, más si se tiene tres o cuatro hijos, ahí si la cosa cambia (E.8).

Desde este argumento se visualiza que la política alimentaria está basando su atención desde la individualidad, pero deja de lado la atención de la inseguridad alimentaria desde un enfoque familiar, que representa una sexta determinante por los vacíos de un proyecto familiar que posibilite la convivencia social, por lo cual las familias que están compuestas por una gran cantidad de miembros no pueden sentarse a comer en el comedor, pues esto significa pagar mucho dinero en una sola comida. Si bien se denota que a partir de que opera el comedor, las personas lo consideran un lugar rápido donde pueden venir y encontrar comida recién hecha; sin embargo, no pueden asistir diariamente, por lo que tener dos visitas a la semana significa un gran reto para el bolsillo familiar. En cuanto a los significados que ha tenido el proyecto en sus vidas, señalan: Está bien cimentando el programa, pero pos no creemos que acabe con el hambre; sirve para mitigarla, para ser una opción "pa" nosotros "pa"algunas ocasiones (E.9).

Desde los argumentos discursivos se habla de poco impacto del programa en su situación alimentaria, ligado a su participación irregular. Para personas que viven de manera aislada o en abandono, como es el caso de adultos mayores, contribuye significativamente, pues asiste y existe la posibilidad de que el alimento sea gratuito. También ha sido significativo para personas que trabajan ahí cerca; representa una opción de comida tal si fuera una lonchería y para el caso de una madre soltera que trabaja y no tiene tiempo de cocinar lleva todos los días al mediodía a sus hijos a comer. Desde el discurso se tiene una política basada en los aspectos culturales del contexto para el fomento de la participación ciudadana, pero desde las mediadoras es contradictoria, pues no se responden a las dimensiones socioculturales de la alimentación.

Por ello, al no contemplar las dimensiones socioculturales del lugar se limita la participación. En esta mirada se encuentra también otra arista importante: la falta de una orientación alimentaria en el momento de echar andar el comedor. Existe un desconocimiento sobre las prácticas alimentarias, las cuales obstruyen el proceso de implementación y la atención 
de la inseguridad alimentaria. Se observa que las personas aceptan que las prácticas alimentarias son un factor sociocultural decisivo a la hora de participar dentro del comedor, este desconocimiento nos refiere falta de un previo diagnóstico para analizar las determinantes socioculturales del contexto.

Se demarca que desde el discurso de los personas beneficiadas se obtienen apreciaciones importantes sobre la política alimentaria, destacando como más que una política para erradicar la situación de inseguridad alimentaria es una política que da respuesta de manera pragmática al hambre, es decir, de forma momentánea, pero no resuelve de forma estructural. Si se busca un cambio, es necesario repensar las vías para atenuar la situación de inseguridad alimentaria. Desde esta visión se observa cómo los programas de alimentación deben partir de las necesidades de los diferentes grupos sociales, de los deseos expresados, del respeto de la identidad cultural. En este sentido, debe existir una orientación alimentaria que contemple los actos de la vida cotidiana, compra, preparación, consumo de alimentos, buscando la incorporación de las recomendaciones a partir de la viabilidad de las mismas.

\section{CONCLUSIONES E IMPLICACIONES}

A pesar de que el programa político en el discurso señala que la política rescata la sabiduría popular de las comunidades, no contempla determinantes socioculturales del consumo y el contexto, los cuales determinan la participación de las personas beneficiadas al programa. Al no responder a las necesidades, los esfuerzos se diluyen, pues no se dispone de diagnósticos previos sobre las prácticas culturales de alimentación, por lo que un programa social no puede aplicarse de un espacio a otro sin sufrir modificaciones, ya que la realidad alimentaria tiene connotaciones diferentes. Asimismo, el programa no va acompañado de orientación alimentaria que permita hibridar las preferencias alimentarias con los alimentos «sanos» que proporcionan en el Comedor Comunitario. Si bien el programa busca atenuar la situación de inseguridad alimentaria, la mayoría de los actores sociales no ha notado cambios significativos en su vida desde la apertura del comedor; considera que es algo propositivo, pero que no abatirá con el problema del hambre.

El programa sigue reproduciendo los esquemas operativos, que se caracterizan por la entrega de satisfactores alimentarios pragmáticos, que si bien se resuelven de manera momentánea, no erradican de manera estructural la situación de inseguridad alimentaria, por lo que siguen siendo prácticas paliativas. En materia de políticas sociales, es im- prescindible la creación de modelos críticos que incorporen la subjetividad de las personas beneficiadas de la política alimentaria, es importante conocer el espacio de significaciones sociales donde se dan las interacciones sociales de los sujetos. Asimismo, recuperaría los elementos culturales, sociales, económicos y políticos que encierran las prácticas alimentarias desde una postura holística.

\section{REFERENCIAS BIBLIOGRÁFICAS}

Alguacil, G., J. (2011). Cómo se hace un trabajo de investigación en sociología. Madrid: Catarata.

Arellano, M., C y Álvarez, M., C. (2019). Aproximación a la coherencia política de los comedores comunitarios de la Cruzada contra el Hambre: Situación en una comunidad agroindustrial en Sonora. RLEEI. Revista Latinoamericana de Educación y Estudios Interculturales 3 (2), 43-55. Recuperado de: https://zenodo.org/badge/DOI/10.5281/ zenodo.2658654.svg

Cabello, M., L. y Amador, S., R. (2015). Entre la escasez, la abundancia y el riesgo alimentario. Propuestas desde el trabajo social En: S. Vázquez, B., G., León y E., Montemayor. (Coords.) Riesgos y Trabajo Social. México: Porrúa, 49-60.

Cabello M., L. y Hernández A., S. (2016). El grupo de ayuda mutua como modelo de intervención en un contexto comunitario. El caso de mujeres con obesidad mórbida. Interacción y Perspectiva. Revista de Trabajo Social. 6 (2), 196-215.

Carrasco, N. (2004). Antropología de los problemas alimentarios contemporáneos, etnografía de la intervención alimentaria en la región de la Arancania. Tesis doctoral. Universidad Autónoma de Barcelona, Facultad de Letras, España.

Consejo Nacional de Evaluación de la Política de Desarrollo Social (CONEVAL) (2015). Medición de la Pobreza en México y en las Entidades Federativas 2014. México: Coneval 2015. Recuperado de: http://www.coneval.org.mx/ SalaPrensa/Documents/Comunicado005_Medicion_pobreza_2014.pdf

Díaz, Z. C. (2007). Habermas: lenguaje y diálogo, el rol del entendimiento intersubjetivo en la sociedad moderna. Utopía y Praxis Latinoamericana, 12 (39), 47- 72. Recuperado de: https://www.redalyc.org/pdf/279/27903904.pdf

Díaz, J. y Candela, Y. (2014). Políticas alimentarias y nutricionales en los sistemas nacionales de salud. AnVenezNutr, 27 (1), 143-152. Recuperado de: https://www.analesdenutricion.org.ve/ediciones/2014/1/art-19/ 
Durham, E. R. (1984). Cultura e ideología. Dados, Revista de Ciencias Sociales, 27, (1). 141-153.

García, C. N. (1989). Culturas hibridas: estrategias para entrar y salir de la modernidad. México: Grijalbo.

Giménez, G. (2005). La concepción simbólica de la cultura. Teoría y análisis de la cultura. México: CONACULTA. 67-80 Recuperado de: https://pics.unison.mx/SemyAct/ LA_CONCEPCION_SIMBOLICA_DE_LA_CULTURA\%$5 b 1 \% 5 d . p d f$

Habermas, J. (1987). Teoría de la acción comunicativa I. Racionalidad de la acción y racionalización social. Madrid: Taurus.

Hurtado, A., M. (2019). Seguridad alimentaria y nutricional, derecho humano a la alimentación adecuada y soberanía alimentaria: una aproximación desde los instrumentos de derecho internacional. La Calera. Revista Científica 19 (32), 55-58. DOI: https://doi.org/10.5377/calera. v19i32.8441

López, R. E. y Deslauriers J. P. (2011). La entrevista cualitativa como técnica para la investigación en Trabajo Social. Margen, (61), 1-19.

Molero, J.; López, D y Arroyo, L. (2018). Salud y Derecho a la Alimentación. Bienestar, equidad y sostenibilidad a través de políticas alimentarias locales. España: Fundación Entretantos y Red de Ciudades por la Agroecología.

Mundo, V.; Shamah-Levy, T. y Rivera-Dommarco, J. A. (2013). Epidemiología de la inseguridad alimentaria en México. Revista Salud Publica en México, 55 (2), 206-213. http:// dx.doi.org/10.21149/spm.v55s2.5117

Organización para la Cooperación del Desarrollo Económico (OCDE) (2017). Health at a Glance 2017: OCDE Indicators. Recuperado de: https://www.oecd.org/mexico/Health-ata-Glance-2017-Key-Findings-MEXICO-in-Spanish.pdf

Organización Mundial de las Naciones Unidas para la Alimentación y la Agricultura (2019). El estado de la segu- ridad alimentaria y la nutrición en el mundo. Protegerse frente a la desaceleración y el debilitamiento de la economía. Recuperado de: http://www.fao.org/3/ca5162es/ ca5162es.pdf

Secretaría de Desarrollo Social (SEDESOL) (2015). Lineamientos específicos del Programa de Comedores Comunitarios en el marco de la Cruzada Nacional Contra el Hambre para el ejercicio fiscal 2015. Recuperado de: http:// www.normateca.sedesol.gob.mx/work/models/NORMATECA/Normateca/1_Menu_Principal/2_Normas/3_Historico/2015/Lineamientos_Prog_Comedores_Comunitarios_23122015.pdf

Sordini, M. V. (2016). La cuestión alimentaria como cuestión social. Los programas alimentarios implementados entre 1983 y 2001 en Mar del Plata, Argentina. Azarbe Revista Internacional de Trabajo Social y Bienestar, (5), 49-58.

Verdugo, L. M. (2012). La política social del programa desayunos escolares desde la perspectiva del trabajo social, caso: primaria Ricardo Flores Magón. Trabajo fin de máster inédito. Universidad Autónoma de Sinaloa, Facultad de Trabajo Social, Culiacán, México.

Verdugo-Araujo, L.M, Tereso-Ramírez, L. y Carrillo-Montoya, T. N.J. (2019). La participación comunitaria como vía para el empoderamiento de encargadas del programa Comedores Comunitarios en Culiacán, México. Prospectiva. Revista de Trabajo Social, 28, 145-168. DOI: 10.25100/prts. v0i28.8052

Yaschine, I., Ochoa, S. y Hernández, C. (2014). Cruzada Nacional Contra el Hambre: análisis de su diseño desde un enfoque de seguridad alimentaria. México: Universidad Autónoma de México. Programa Universitario de Estudios del Desarrollo. Recuperado de: http://www.pued. unam.mx/export/sites/default/archivos/documentos-trabajo/010.pdf 